\title{
VirCA NET: A Case Study for Collaboration in Shared Virtual Space
}

\author{
Péter Galambos ${ }^{* \dagger}$, Christian Weidig ${ }^{\S}$, Péter Baranyi ${ }^{* \ddagger}$, Jan C. Aurich ${ }^{\S}$, Bernd Hamann ${ }^{\mathbb{I}}$, Oliver Kreylos ${ }^{\mathbb{I}}$ \\ * Computer and Automation Research Institute, Hungarian Academy of Sciences, Budapest, Hungary \\ $\dagger$ Department of Manufacturing Science and Technology, Budapest University of Technology and Economics \\ ‡Department of Telecommunications and Media Informatics, Budapest University of Technology and Economics \\ $\S$ Institute for Manufacturing Technology and Production Systems, University of Kaiserslautern, Germany \\ II Institute for Data Analysis and Visualization (IDAV), University of California, Davis, USA \\ E-mail: \{galambos, baranyi\}@sztaki.hu, \{weidig, aurich\}@cpk.uni-kl.de, \\ \{hamann, kreylos\}@cs.ucdavis.edu
}

\begin{abstract}
To take up the challenge of global distributed companies, Manufacturing Engineering has to be accomplished in a worldwide collaborative way. Design and development of production processes and their execution are nowadays spread all over the world. To ensure quality standards and to create synergies between spatially distributed entities, distance collaboration tools must be provided to allow cooperation even over large distances. The Virtual Reality (VR) is thereby offering beneficial capabilities to exchange current planning stages, identify problems and solve them cooperatively. This paper introduces a new approach for distance collaboration, which enables users to work in a joint virtual space, nearly as if they were working together in the same place. Therefore two full-immersive CAVE-like systems are interconnected using the VirCA (Virtual Collaboration Arena) platform. The paper describes the requirements of Mechanical Engineers towards distance collaboration tools and the technical challenges solved with the enhanced VirCA framework. This working prototype is one premier application in the field of VRenhanced spatially distributed collaboration. A typical use case scenario is provided to highlight the interaction and cooperation capabilities offered by VirCA NET.
\end{abstract}

Index Terms-3D Internet, Virtual reality, Augmented reality, Collaboration platform, Factory layout design, Digital factory

\section{INTRODUCTION}

Manufacturing Engineering is facing with the continuous adaptation of factories regarding changing requirements of markets, supply networks and emerging product complexity. Simultaneously economic efficiency must be ensured [1]. Thereby the inclusion of multiple stakeholders to the adaptation processes along the factory life cycle is crucial. Several divisions and employees must be involved, to avoid nonoptimal planning and exploit their special planning expertise and profession [2]. This task gets even more complex, if spatially distributed entities are involved. Nevertheless tailoring of production systems according different local constraints is required to fulfil the demands of worldwide markets, respect the cultural diversities and consider existing local conditions. To overcome this challenge the support of manufacturing engineering by means of collaboration, to handle the key characteristics of spatially distributed but synchronous planning is proposed [3], [4]. Especially for multinational corporations, operating multi-site production systems, supporting means offered by VR should be taken into account. Configuration and reconfiguration processes of flexible production factories require features especially for supporting multi stakeholder decision making and joint validation of planning results [5].

Hence factory planning in detail is identified as one keydomain applicable for collaborative VR and Augmented Systems. The capability to design real environments by the use of virtual objects and the integration of human intuitiveness make this field predestined to be enhanced by VR tools. The proposed main collaborative tasks can be summarized as modelling of the factory, validation of the planning stage and subsequent optimization of the production system [6]. This is coherent with [7] who stated that often the most effective way to describe, explore, and summarize a set of numbers is to look at the picture of those numbers. Therefore the distance collaboration approach introduced in this paper will focus on the simultaneous usage of a shared virtual environment. This enables simultaneous investigation of the same virtual model by spatially distributed users. The approach is beneficial as it fosters the existing VR-enhanced work-flow of mechanical engineers for factory planning tasks. Users are allowed to work within their used processes and known problem solving strategies, but extended to the distance collaborative aspect [8].

The proposed approach has several aspects closely related to CogInfoCom [9], therefore, this topic is discussed separately in [10].

The paper is structured as follows: Section II discusses the end-user requirements and defines the crucial points on which the development of distant collaboration tools must be focused. Section III introduces the VirCA NET framework that partially fulfils the previously uncovered requirements. Section IV deals with the implementation of a realistic use case scenario where the main objective is to realize the collaborative planning of a manufacturing cell consisting of a machining centre and a robot manipulator as main equipments. Finally, Section V summarizes the paper. 


\section{MAnUfacturing EngINEERING REQUiREMENTS}

To grasp and fulfil the user requirements on VR distance collaboration tools for factory planning, the following section will describe three main requirements coming from the field of mechanical engineering. Derived from the initially sketched situation which factory planning is faced with the following features are identified as crucial:

1) shared model visualization

2) model interaction

3) human interaction, knowledge exchange

The 3D virtual model of the production system is the base on which adaptations of the shop floor are planned, analysed and evaluated within a collaborative session. Therefore the digital model consists of geometric descriptions of objects (environment and manufacturing related) and additional information from the production system, dealing with process descriptions for example [11]. Sharing this virtual model with all planning participants and allowing a comprehensive, distinct view for all users is a key requirement of a distance collaboration tool [3]. VirCA NET is tackling this objective by providing a joint shared virtual environment, which appearance is identical at all remote sites.

In addition to the shared visualization of factories, interaction with the virtual world must be integrated into VirCA NET. The understanding of production processes and the investigation of simulation results out of the virtual environment is facilitated by bidirectional interaction with the virtual model. On the one hand, there are collaborative features for interaction between users and virtual agents, but there are interconnections between several virtual agents as well. The major objective of distance collaborative tasks is not to develop a new model or product. Rather investigating models and solving problems cooperatively is focused. Accordingly, cognitive features must be integrated into the distance collaboration tool; social interaction metaphors beyond the data visualization must be provided [12], [13]. Hence one objective is to support knowledge transfer between remote sites in a social oriented way. In detail, knowledge related to personal skills, that is not graspable formerly but tacit knowledge which is hardly transferable without direct personal interaction, must be considered [14]. Therefore human-human interaction means must be integrated into the VirCa NET.

Concluding these requirements a typical application field for VirCA NET can be sketched up in the scope of factory layout planning. The core idea is to transfer a well-known working situation, namely the co-located domain-overlapping planning into a shared virtual environment. Spatially distributed planning partners should work together as if they were in-place (discuss, introduce problems, exchange points of view, find solutions and so on). The model will allow a simultaneous investigation, while users chose their focus and point of interest by their own. The distributed intelligent agents are influencing each other and user actions will be transmitted between each remote sites. The interconnected VR systems will react as one entity and users should have the impression as if they were co-located like in the so-called FPS computer games played in network mode. Augmenting this technical setup by social human-human interaction capabilities will complete the perception of direct collaboration. This is beneficial since the exchange of implicit knowledge is simplified and supported by direct personal contact between planning partners. Due to close correlation to known working situations, discussions among a digital model of the current planning stage should lead to the same optimization as if developed in a co-located scenario.

\section{THE VIRCA NET FRAMEWORK}

In this section, the VirCA NET framework is introduced focusing on the previously discussed requirements. Virtual Collaboration Arena (VirCA) is a software platform for component-based applications wherein the components are reusable and can be running at different locations on the Internet supporting the geographically distributed system design. An introduction to VirCA can be found in [15] where it is discussed from the aspect of the Intelligent Space concept [16]. The VirCA framework incorporates the following three underlying functionalities:

- Immersive or non-immersive 3D visualization of a shared virtual world

- Unified communication middleware to involve different kind of devices and knowledges into the scene

- Web-based system manager to build and manage complex systems from reusable components composing the VirCA NET scenarios

VirCA NET is an extension of the VirCA platform where multiple instances of VirCA are interconnected making an advanced collaboration possible between remote users who become able to design, simulate and operate complex systems (such as factories) in a shared virtual reality.

\section{A. Basic concepts}

The basic technical concepts related to VirCA NET are defined here:

\section{Collaborator}

In VirCA NET context, Collaborator is understood as a person or a group of persons who are interacting with the shared virtual reality at the same location. For example a team of 4 persons working in the CAVE system at University of Kaiserslautern considered as one Collaborator of the VirCA NET session.

\section{Master VirCA}

The VirCA instance where the other VirCA instances are connected to. Master VirCA acts as a hub for the interconnected VirCAs.

\section{Slave VirCA}

A VirCA instance that is connected to the Master VirCA. Multiple slaves can be connected to a single master. Slave VirCA instances are cloning the virtual space maintained by the master VirCA.

VirCA NET connection

The communication channel between the master 
VirCA and a slave VirCA. This channel is responsible for the synchronisation of the virtual space and for the transmission of user actions in order to ensure that all collaborators receives the same information from the shared virtual reality.

RT-Component or RTC for short

A reusable software component that complies the Robot Technology Component Specification [17]. Different type of capabilities (e.g. machine vision, balancing, etc.) and hardware interfaces (e.g. sensors, actuators, complex devices) can be implemented in form of RTCs. In VirCA context, RTC is a reusable building block that is directly not appears in the virtual space but operates as background knowledge or interfacing the virtual and real-world objects.

Cyber Device or CD for short

Cyber Devices are special type of RTCs which appears as 3D objects in the shared virtual space. Machine tools, robots and other visually manifested parts of the production system are implemented in form of Cyber Devices.

Input Device

Input devices are software components interfacing different UI hardware to VirCA (e.g. pointing devices, MS Kincet, etc.).

System Editor

VirCA System Editor is a web application for the management of the collaboration session. Collaborators can build the system from Input Devices, RTCs and CDs as building blocks.

For more details, please consult the documentation available at the VirCA website [18].

\section{The CAse Study}

In this section, we introduce a concrete use case scenario that illustrates the capabilities of the VirCA NET framework.

\section{A. The Scenario}

For the purpose of pilot tests we made up a realistic scenario that covers some essential issues of collaborative factory planning. In this story, a manufacturer company prepares the installation of a new manufacturing cell composed by a machining centre, a robot and storage equipments on an empty shop-floor. The company as procurer is in contact with a machine tool supplier and a robotic system integrator specialist in order to select, test and purchase the proper equipments. These three partners are considered as collaborators in the planning project. They should come to decision on the sort of the machines, the exact location of the machines within the shop and they should test the whole manufacturing cell in operation. The test should be performed in a semi-real semivirtual manner, which means that the whole system is built up and operated virtually in VirCA NET while some of the system elements (e.g. the robot) are working in reality in parallel to its virtual counterpart.

\section{B. Implementation Details}

The before described scenario is incorporates three VirCA instances according to the three stakeholders. Figure 1 shows a possible build-up to realize the collaborative session. The procurer company (LOCATION 1) hosts the master VirCA and the cell controller Cyber Device, while the robot and machine tool supplier companies (LOCATIN 2 and LOCATION 3) are running the slave VirCA instances and the components related to the devices to be tested.

Slave VirCA instances are connected to the master VirCA via VirCA NET interfaces that ensures the synchronisation of the distributed 3D scene. Each collaborators are responsible only for the maintenance of those components that are related to their special expertise. It means that the robot supplier runs a Robot CD and a Robot RTC while the machine tool dealer provides the machine tool $\mathrm{CD}$ and the corresponding RTC. CDs are responsible only for the visualisation of the devices, while the RTCs cover the functionalities of the machine or robot controllers. It must be mentioned here that the communication between the cell controller and the machine tool or robot controller RTCs should be managed using standard generic interfaces that are not matured enough when this paper is written. Generalisation of interfaces for component based systems and interoperable devices are under intensive research [19], [20], [21] and addressed by several projects [22], [23].

Figure 2 illustrates the shared scene from the viewpoint of a collaborator. This screen shot displays the shop floor where the robot should be placed in accordance with the decision of the collaborators.

\section{Discussion}

VirCA NET provides shared visualization and supports several way of interaction between the components and the collaborators involved in the cooperative session. However, the recent version of the software provides only a limited set of features addressing human-human communication. For example, within the virtual world, collaborators can only see each other as a symbolic human head representing the gaze direction. To establish voice and video connection other software tools must be used simultaneously. Such integrated features will be the target of further development of the VirCA NET framework.

An other crucial point is the way of manipulation of virtual objects. Since users should not be burdened with having to wear complicated haptic devices, CogInfoCom methods takes place that could provide unencumbered yet situation aware user experience [24].

Because of its extensible modular structure, the proposed scenario serves as good basis for the experimental investigation of different interaction methods.

\section{SUMMARY}

In this paper, a Virtual Reality based multi-user remote collaboration was studied considering a factory planning use case scenario. The requirements against the collaboration system were discussed from the viewpoint of manufacturing engineers 


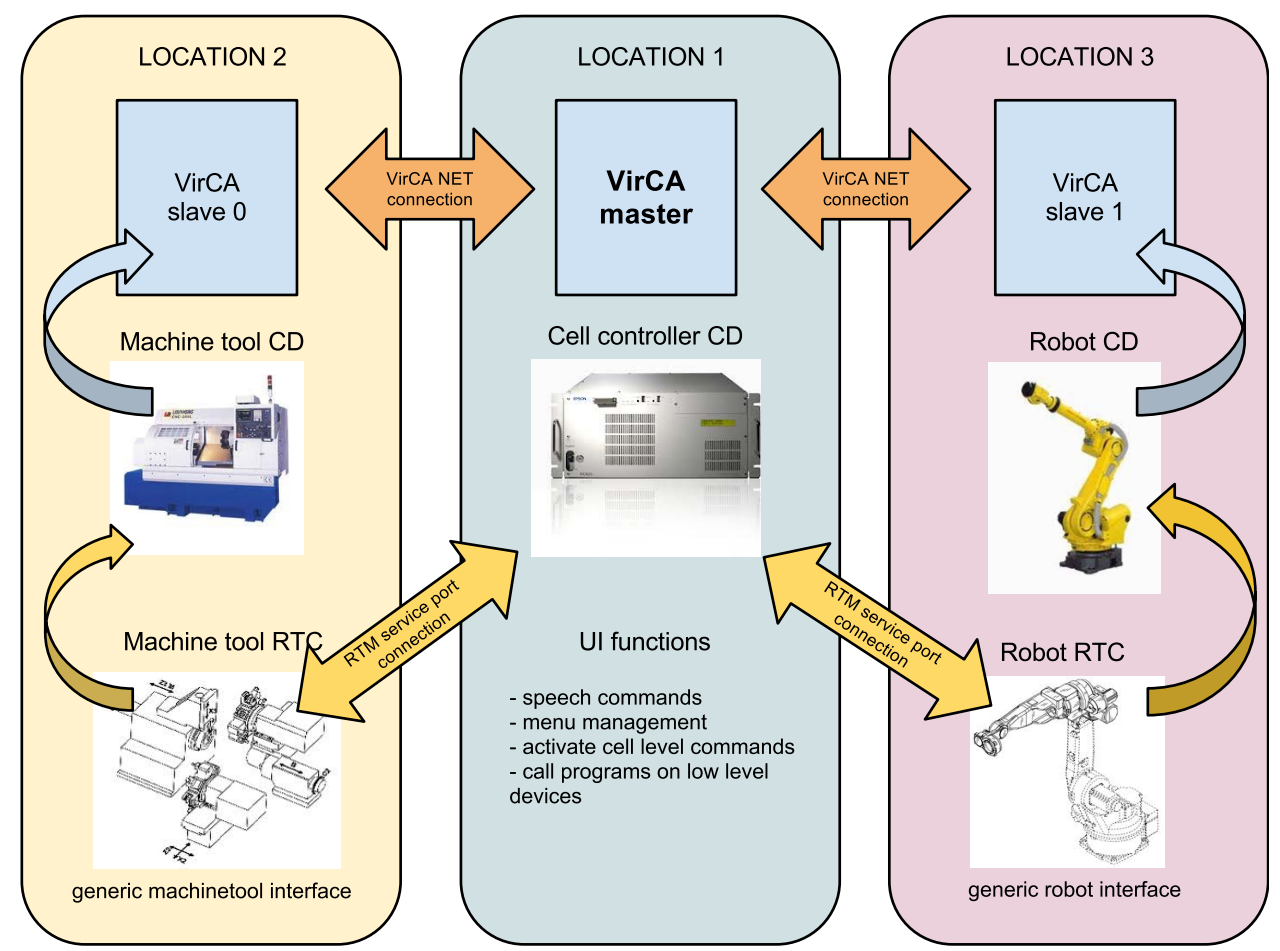

Fig. 1. The VirCA NET structure of the proposed collaborative planning session

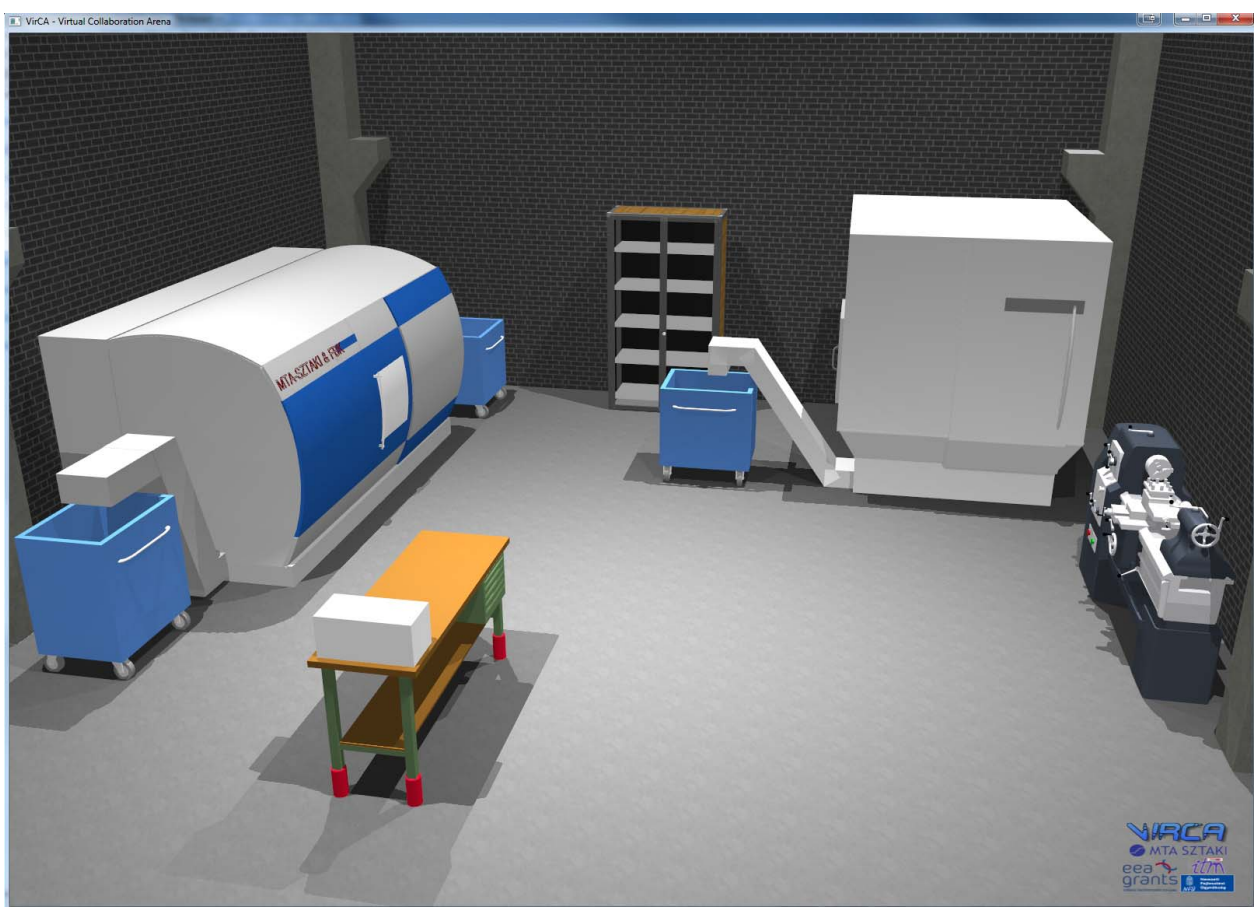

Fig. 2. A screen shot from the viewpoint of a collaborator

as end-users, then the VirCA NET platform was introduced focusing on its capabilities that fulfil these requirements. A concrete example for the collaborative design of a small manufacturing cell was investigated and the technical details of the implementation were discussed. Via this use-case study it is shown that the VirCA NET approach can be beneficially utilized in the investigated scenario wherein the component selection, the shop floor layout design and process simulation/evaluation are integrated in one versatile collaboration system. On the other hand, the human-human communication 
capabilities and the way of manipulating virtual objects must be enhanced. The proposed approach and the pilot application allow the community to implement and investigate different CogInfoCom channels in a real-life scenario.

\section{ACKNOWLEDGMENT}

The research leading to these results has received funding from the European Community's Research Infrastructure Action - grant agreement VISIONAIR 262044 - under the 7th Framework Programme (FP7/2007-2013).

\section{REFERENCES}

[1] E. Westkämper, C. Constantinescu, and V. Hummel, "New paradigm in manufacturing engineering: Factory life cylce," Production Engineering Research and Development, vol. 13, no. 1, pp. 143-146, 2006.

[2] B. Denkena, P. Woelk, and A. Brandes, "Flexible process chains by template based configuration," Annals of the German academics society for production engineering, vol. 12, no. 2, pp. 81-84, 2005.

[3] N. Menck, X. Yang, C. Weidig, P. A. Winkes, C. Lauer, H. Hagen, B. Hamann, and J. C. Aurich, "Collaborative factory planning in virtual reality," in The 45th CIRP Conference on Manufacturing Systems, Athens, Greece, May 2012.

[4] T. Liebeck, T. Meyer, and E. Abele, "Production technology: Adapting to maximize local advantage," Global Production: a handbook for strategy and implementation, Springer, Berlin, pp. 192-235, 2008.

[5] P. Pedrazzoli, D. Rovere, C. Constantinescu, J. Bathelt, M. Pappas, P. Depince, G. Chryssolouris, C. R. Boer, and E. Westkämper, "High value adding vr tools for networked customer-driven factory," in Proceedings of the 4th International Conference on Digital Enterprise Technology, Bath, United Kingdom, 2007.

[6] A. Nee, S. Ong, G. Chryssolouris, and D. Mourtzis, "Augmented reality applications in design and manufacturing," CIRP Annals - Manufacturing Technology, no. 61, pp. 657-679, 2012.

[7] E. Tufte, The Visual Display of Quantitative Information, 1st ed. Graphics Press, 1983.

[8] O. Kreylos, G. Bawden, T. Bernardin, M. I. Billen, eric S. Cowgill, R. D. Gold, B. Hamann, M. Jadamec, L. H. Kellogg, O. G. Staadt, and D. Y. Sumner, "Enabling scientific workflows in virtual reality," in Proceedings of the ACM International Conference on Virtual Reality Continuum and Its Applications, Hong Kong, 2006, pp. 155-162.

[9] P. Baranyi and A. Csapo, "Definition and Synergies of Cognitive Infocommunications," Acta Polytechnica Hungarica, vol. 9, pp. 67-83, 2012.

[10] C. Weidig, A. Csapo, J. C. Aurich, B. Hammann, and O. Kreylos, "Vircanet and coginfocom: Novel challenges in future internet based augmented/virtual collaboration," in 3rd IEEE International Conference on Cognitive Infocommunications, Kosice, Slovakia, dec 2012.

[11] X. Yang, R. C. Malak, C. Lauer, C. Weidig, H. Hagen, B. Hamann, and J. C. Aurich, "Virtual reality enhanced manufacturing system design," in Proceedings of the 7th CIRP International Conference on Digital Enterprise Technology, Athens, Greece, sept 2011, pp. 125-133.

[12] D. Russell, M. Stefik, P. Pirolli, and S. Card, "The cost structure of sensemaking," in Proceedings of the Conference on human Factors in Computing Systems (CHI), Amsterdam, The Netherlands, april 1993, pp. 269-276.

[13] P. Isenberg, N. Elmqvist, J. Scholtz, D. Cernea, K.-L. Ma, and H. Hagen, "Collaborative visualization: Definition, challenges, and research agenda," Information Visualization, vol. 10, no. 4, pp. 310-326, 2011.

[14] Y. Cheng, E. S. Madsen, and J. Liangsiri, "Transferring knowledge in the relocation of manufacturing units," Strategic Outsourcing: An International Journal, vol. 3, no. 1, pp. 5-19, 2010.

[15] P. Galambos and P. Baranyi, "Virca as virtual intelligent space for rt-middleware," in Advanced Intelligent Mechatronics (AIM), 2011 IEEE/ASME International Conference on, july 2011, pp. $140-145$.

[16] H. Hashimoto, "Intelligent space: Interaction and intelligence," Artificial Life and Robotics, vol. 7, pp. 79-85, 2003.

[17] Robot technology component specification. [Online]. Available: http://www.omg.org/spec/RTC/

[18] MTA SZTAKI, VirCA (Virtual Collaboration Arena). [Online]. Available: http://www.virca.hu
[19] P. Iñigo-Blasco, F. Diaz-del Rio, M. Romero-Ternero, D. CagigasMuñiz, and S. Vicente-Diaz, "Robotics software frameworks for multiagent robotic systems development," Robotics and Autonomous Systems, 2012.

[20] H. Wei, J. Lei, and T. Wang, "A kind of architecture and key technologies for developing modular robot," Advances in Reconfigurable Mechanisms and Robots I, pp. 561-568, 2012.

[21] M. Tenorth, A. Perzylo, R. Lafrenz, and M. Beetz, "The roboearth language: Representing and exchanging knowledge about actions, objects, and environments," in Robotics and Automation (ICRA), 2012 IEEE International Conference on. IEEE, 2012, pp. 1284-1289.

[22] RoboEarth project. [Online]. Available: http://www.roboearth.org/

[23] BRICS project. [Online]. Available: http://www.best-of-robotics.org/

[24] P. Galambos, "Vibrotactile feedback for haptics and telemanipulation: Survey, concept and experiment," Acta Polytechnica Hungarica, vol. 9, no. 1, pp. 41-65, 2012. 